\title{
EDITORIAL
}

\section{México sin tabaco}

$\mathbf{E}^{\prime}$ 1 creciente consenso mundial acerca de las mejores prácticas para enfrentar la epidemia del tabaquismo se cristalizó en el Convenio Marco para el Control del Tabaco (CMCT) y México fue uno de los primeros países en ratificarlo. Como su nombre lo indica, el CMCT es un referente global para la acción y ha conferido impulso y fundamento a la aplicación de leyes eficaces.

La legislación es la base de un control eficiente del consumo de tabaco. Expresa valores profundamente enraizados en la sociedad, institucionaliza el compromiso de un país, centra las actividades y regula el comportamiento privado. Sin embargo, la promulgación de una legislación sólida supone retos difíciles. Amenudo existe un limitado conocimiento público del problema y también se pone a prueba la capacidad nacional, es decir, la infraestructura y los recursos para infundir un apoyo decisivo. El principal obstáculo para que opere el CMCT es la oposición de la industria tabacalera y las atractivas alternativas que ésta puede ofrecer. En este sentido, no podemos perder de vista jamás que el único objetivo del control del tabaco es la reducción del consumo de cigarros y otros productos del tabaco.

La capacidad institucional de un país para respaldar los esfuerzos de control del tabaco es la base del éxito para aplicar las medidas que sugiere el CMCT. El fortalecimiento de esa capacidad cambia la cultura de la sociedad, que pasa de aceptar como normal el consumo de tabaco, a rechazarlo como desagradable, adictivo y nocivo para ella. Los temas identificados como medulares en una legislación integral para el control del tabaco son, según la Organización Mundial de la Salud, ${ }^{1}$ los siguientes:

Incrementar los impuestos. Se ha demostrado que el aumento de los impuestos es uno de los medios más eficaces para atenuar el consumo de tabaco, sobre todo entre los jóvenes.

Regular la venta de tabaco. La legislación debe prohibir la venta de tabaco a los menores y prevenir modalidades de venta perjudiciales, por ejemplo mediante distribuidores automáticos y exhibición en autoservicios.
Proteger el ambiente del humo de tabaco. La eliminación del humo de tabaco en los sitios de trabajo y lugares públicos protege a quienes no fuman de los riesgos para su salud, desalienta el inicio del tabaquismo y promueve el abandono del hábito.

Promover la cesación. Las medidas para alentar en los fumadores el abandono del cigarro son una parte crítica del abordaje integral y complementan las medidas centradas en la educación y la prevención.

Prohibir la publicidad, promoción y patrocinio. La prohibición integral de la publicidad, la promoción y el patrocinio del tabaco es un factor esencial de un programa basado en la evidencia.

Proporcionar educación pública. Las grandes campañas de educación del público son importantes para cambiar las creencias y actitudes.

Instituir programas escolares. Es muy importante incluir en los programas escolares información relevante sobre los daños que causa fumar. Dado que el consumo de tabaco empieza a edades cada vez más tempranas, la enseñanza de los daños a la salud también debe comenzar desde los primeros niveles.

Regular el etiquetado y empaquetado. Debe exigirse que el empaquetado de los productos del tabaco incluya advertencias sanitarias grandes y claras e información en forma de mensajes rotativos establecidos por las autoridades nacionales. Debe prohibirse por completo el uso de términos engañosos, como ligeros (light) o suaves (mild).

Reglamentar los productos. Se debe otorgar autoridad normativa a un organismo especializado, encargado de asuntos como la divulgación de información sobre ingredientes, la permisividad de los componentes nocivos, la inocuidad de los aditivos y el contenido de alquitrán y nicotina.

Combatir el contrabando. Para enfrentar el comercio ilícito, la legislación integral debe incluir diversas medidas, entre ellas exigir requisitos sobre el marcado de los paquetes o crear un sistema de seguimiento y localización de los productos a lo largo de la cadena de distribución. 
Si bien cada uno de los elementos descrito es importante, más útil resulta considerarlos como componentes de una política coordinada; de esa manera puede conseguirse un efecto sinérgico y delinear un programa integral. Este tipo de intervenciones conjuntas sólo puede promoverlo el gobierno, que al ratificar el CMCT fijó su postura sobre este tema. Sin la intervención gubernamental es imposible llegar a los diferentes grupos poblacionales y reducir en consecuencia las inequidades. ${ }^{2}$

Para lograr el cambio de conductas individuales que se opongan al consumo de tabaco, la sociedad debe cambiar la forma en que éste se promueve, vende y consume. El cigarro y la exposición al humo incrementan los riesgos de las personas a sufrir varias afecciones; por lo tanto, el tema de la cesación se debe analizar en el contexto más amplio de la prevención de enfermedades.

Los programas de cesación que asisten de forma exitosa a jóvenes y adultos pueden tener beneficios rápidos y a largo plazo, más que otros elementos de un programa integral de control del tabaco. Las personas que dejan de fumar reducen sus riesgos de morir, así como los costos de atención médica de enfermedades futuras. Es por estas razones que lograr y promover un consumo cada vez menor es el camino inequívoco para controlar el tabaco.

Hay suficiente evidencia para sostener que los programas escolares que identifican las influencias sociales en el inicio del consumo de tabaco, y que enseñan habilidades para resistir tales influencias, reducen en grado significativo el consumo, o al menos, retrasan la edad de inicio. Por ello, las intervenciones educativas contra el uso del tabaco y otras drogas deben comenzar en la primaria y continuar en la educación media y media superior.

Velar por el cumplimiento de las políticas de control del tabaco es otro gran reto. La vigilancia y evaluación sistemática influyen de forma positiva en dos sentidos: primero, diluyen las intenciones de violar la reglamentación y, segundo, envían un mensaje inequívoco a la comunidad: las políticas son importantes. La consecución de lo anterior representa una gran diferencia en relación con el acceso de los menores a los productos de tabaco y la protección de los no fumadores. Otras áreas importantes son la restricción a la publicidad y toda forma de promoción de los productos del tabaco.

Cada 31 de mayo, como ha sucedido desde hace varios años, el Instituto Nacional de Salud Pública (INSP) pone a disposición de la comunidad científica nacional e internacional, de las instituciones y profe- sionales interesados en el control del tabaco y, sobre todo, del pueblo de México los conocimientos actualizados acerca del control y la prevención del tabaquismo. Una vez más, autores nacionales y extranjeros de reconocidas instituciones se han reunido en una producción editorial del INSP para presentar los resultados, avances y retos que plantea el control del tabaco. Para gran satisfacción de quienes hemos preparado este material, vemos que es amplio el índice de temas contenidos en este número especial de Salud Pública de México y que se han incluido todos los aspectos antes mencionados que resultan esenciales para el control del tabaco.

México, como primer país del hemisferio occidental que ratificó el $\mathrm{CMCT}$, entró a una nueva etapa al aceptar el compromiso de reforzar la legislación nacional para regular el tabaco. Es cierto que en nuestro país los pasos para combatir al tabaco y reducir su efecto negativo en la salud habían comenzado antes de la aprobación del documento, pero no menos cierto es que existe la necesidad de profundizar las acciones emprendidas y sobre todo velar por su cumplimiento.

Los pasos que se han dado en los últimos años han sido y serán decisivos para enfrentar la epidemia del tabaquismo en México en el siglo XXI. De modo sinóptico es posible asegurar que desde el año 2000 se han instituido las siguientes medidas: incremento considerable del impuesto a los cigarros al igualarse la tasa impositiva de los cigarros con y sin filtro; eliminación de la publicidad de los medios electrónicos e introducción de algunas reformas en cuanto a la publicidad en exteriores y los mensajes de salud en las cajetillas; intervenciones educativas en las escuelas de todos los estados y creación de numerosas clínicas de cesación; y aplicación de la cuarta Encuesta Nacional de Adicciones. Además, apareció el Primer Reporte del Secretario de Salud sobre el Control del Tabaco en México y se consolidó la aplicación de la Encuesta Mundial sobre Tabaquismo en Jóvenes como instrumento de vigilancia del inicio de consumo en la adolescencia.

Todos esos avances, así como los retos que tenemos por delante, deben reforzar nuestro entusiasmo y convicción de que un México sin tabaco es posible y, sin duda, mejor.
Raydel Valdés Salgado*

Dr. Mauricio Hernández-Âvila ${ }^{\ddagger}$

\footnotetext{
* Department of Epidemiology. Johns Hopkins Bloomberg School of Public Health, EUA. Investigador del Centro de Investigación en Salud Poblacional, INSP, México.

‡ Director del Instituto Nacional de Salud Pública, México.
} 


\section{Referencias}

1. World Health Organization. Tools for advancing tobacco control in the $\left.X X\right|^{\text {st }}$ century. Tobacco Control Legislation: an introductory guide. Blanke DD, ed. Geneva, Switzerland:WHO, 2003.
2. Centers for Disease Control and Prevention. Best Practices for Comprehensive Tobacco Control Programs-August 1999. Atlanta GA: US Department of Health and Human Services, Centers for Diseases Control and Prevention, National Center for Chronic Disease

Prevention and Health Promotion, Office on Smoking and Health, August, 1999. 